\title{
Nonlinear laser lithography for indefinitely large- area nanostructuring with femtosecond pulses
}

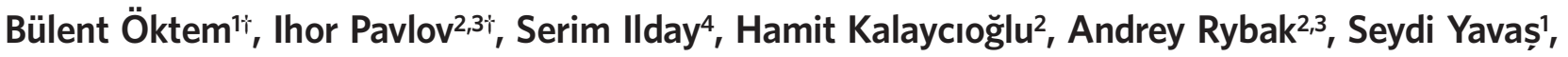 \\ Mutlu Erdoğan ${ }^{1}$ and F. Ömer Ilday ${ }^{2 \star}$
}

Dynamical systems based on the interplay of nonlinear feedback mechanisms are ubiquitous in nature ${ }^{1-5}$. Well-understood examples from photonics include mode locking ${ }^{6}$ and a broad class of fractal optics ${ }^{7}$, including self-similarity ${ }^{8}$. In addition to the fundamental interest in such systems, fascinating technical functionalities that are difficult or even impossible to achieve with linear systems can emerge naturally from them ${ }^{7}$ if the right control tools can be applied. Here, we demonstrate a method that exploits positive nonlocal feedback to initiate, and negative local feedback to regulate, the growth of ultrafast laser-induced metal-oxide nanostructures with unprecedented uniformity, at high speed, low cost and on non-planar or flexible surfaces. The nonlocal nature of the feedback allows us to stitch the nanostructures seamlessly, enabling coverage of indefinitely large areas with subnanometre uniformity in periodicity. We demonstrate our approach through the fabrication of titanium dioxide and tungsten oxide nanostructures, but it can also be extended to a large variety of other materials.

The fabrication of nanostructures on surfaces is of paramount importance in nanotechnology and materials science ${ }^{9}$. There are several established techniques, including photolithography, electron-beam lithography, imprint lithography ${ }^{10}$ and laser interference lithography ${ }^{11}$, as well as non-conventional approaches such as selfassembly $^{12}$ and direct laser writing ${ }^{13}$. These techniques require either high-cost, complex systems or offer limited flexibility. An alternative flexible and potentially very low-cost method is laserinduced periodic surface structuring (LIPSS). The first observation of LIPSS dates back to $1965^{14}$. However, after almost 50 years and a large body of published work that has demonstrated LIPSS on various metals, semiconductors and glasses ${ }^{15-19}$, the method has not found widespread use due to the stubborn problem of quality control ${ }^{18,19}$.

Despite the evident role of self-assembly in the LIPSS process, uniformity and long-range order remain poor, a problem we identified as originating from the fact that the structures are initiated from multiple seed locations concurrently and independently, thereby producing an irregular pattern. Because the process is irreversible, without self-correction, these irregularities become frozen. Our solution to this relies on carefully exploiting feedback mechanisms to tightly regulate the formation of nanostructures induced by ultrashort pulses. This process can be summarized in three steps. (1) The laser beam, with a peak intensity close to the ablation threshold for titanium, is focused on a titanium surface, where it is scattered by existing nanostructures or any surface defects ${ }^{15}$. The interference of the scattered and incident fields leads to intensity variations in the immediate neighbourhood of the scattering point. (2) At points where the threshold intensity for ablation is exceeded, titanium reacts rapidly with $\mathrm{O}_{2}$ from the air, forming titanium dioxide $\left(\mathrm{TiO}_{2}\right)$. The use of ultrashort pulses is necessary to ensure this process occurs faster than heat diffusion, as this can smear out the nanometre-scale localization of the deposited laser energy. The first two steps constitute a positive feedback loop (Fig. 1a). As the nanostructure grows, so does its scattering power. (3) The growth mechanism also has an imbedded negative feedback loop. As $\mathrm{TiO}_{2}$ grows on top of the titanium, penetration of $\mathrm{O}_{2}$ through the oxide layer decreases exponentially, decelerating and eventually halting the growth process (Fig. 1b).

The experimental set-up (Fig. 2 and Methods) consists of an ultrafast fibre laser ${ }^{20}$ coupled to a microscope system for real-time observation of the nanofabrication process. All experiments were guided by a semi-phenomenological theoretical model developed by us. The main features of the model are summarized in the following and in the Methods, and the details are discussed in the Supplementary Information. Scattering of the incident laser field from a single point is modelled as dipole radiation ${ }^{15,16}$, with the relative height of the surface point setting the scattering amplitude. This is confirmed experimentally (Fig. 3a) and numerically (Fig. 3b) by the structure formed around an isolated scatterer. The polarization of the laser sets the dipole radiation pattern, which results in regularly spaced nanolines parallel to the laser polarization. Circular polarization, which can be visualized as rotating linear polarization, results in an array of nanocircles. The period of the structures ranges between 600 and $900 \mathrm{~nm}$, depending on the film thickness. Because the film is much thinner than the wavelength of light, light experiences a sort of a weighted average (effective) index of refraction which depends not only on that of the thin film, but also on those of the air and the substrate above and below the film, respectively. The total field at any surface point is the sum of the incident field and the total scattered field, which is given by the integral of the product of the surface height and the incident field over the entire surface. This surface integral is the mathematical origin of the nonlocal feedback. The amplitude of the dipole radiation decays with distance, which sets a finite range for this nonlocal feedback, such that two distant points on the surface have negligible mutual influence. For this reason, processing a large area at once results in structures with poor long-range order, as seen experimentally (Fig. 3c) and numerically (Fig. 3d). By limiting the size of the laser beam to $\lesssim 10$ wavelengths, we ensure that even the most distant points under the beam have contributions to their mutual fields. This way, the problem of independent structure initiation is solved.

At points where the total intensity exceeds the ablation threshold $\left(\sim 1 \times 10^{12} \mathrm{~W} \mathrm{~cm}^{-2}\right)$, the metal (titanium) disassociates from the solid phase under the non-equilibrium conditions created by the ultrashort pulse and reacts with $\mathrm{O}_{2}$ from the ambient atmosphere,

'UNAM-Institute of Materials Science and Nanotechnology, Bilkent University, 06800 Ankara, Turkey, ${ }^{2}$ Department of Physics, Bilkent University, 06800 Ankara, Turkey, ${ }^{3}$ Institute of Physics, National Academy of Science of Ukraine, Kiev, Ukraine, ${ }^{4}$ Department of Micro and Nanotechnology, Middle East Technical University, 06800 Ankara, Turkey; †These authors contributed equally to this work. *e-mail: ilday@bilkent.edu.tr 
a
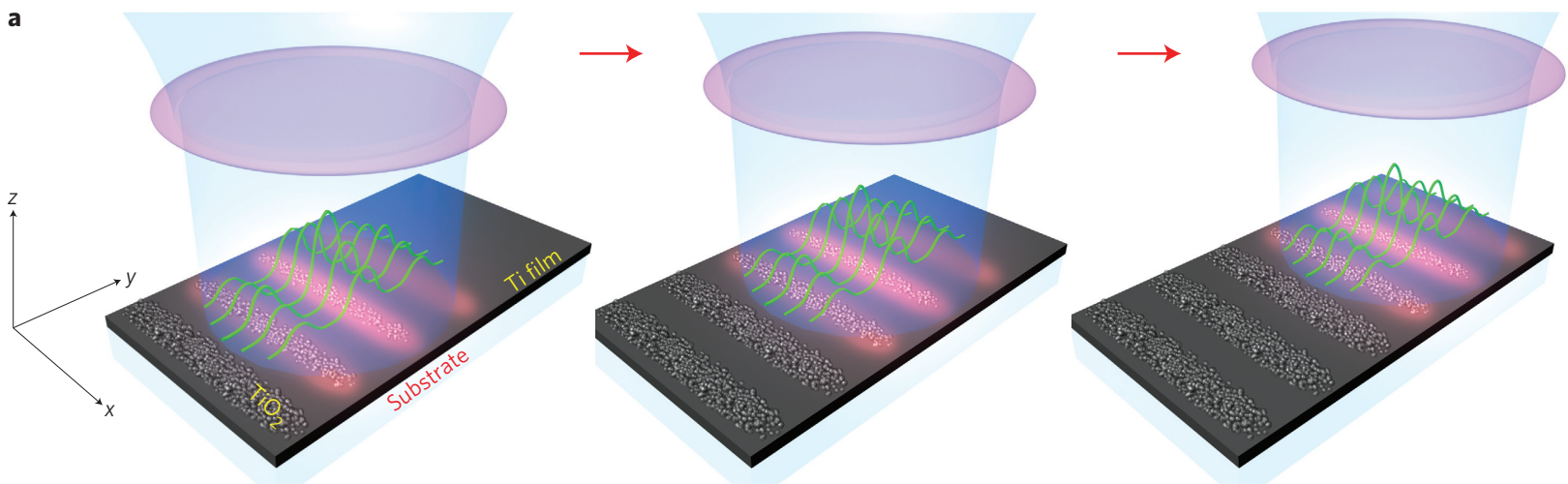

b

$\mathrm{O}_{2}$

$\mathrm{O}_{2}$

$\mathrm{O}_{2}$
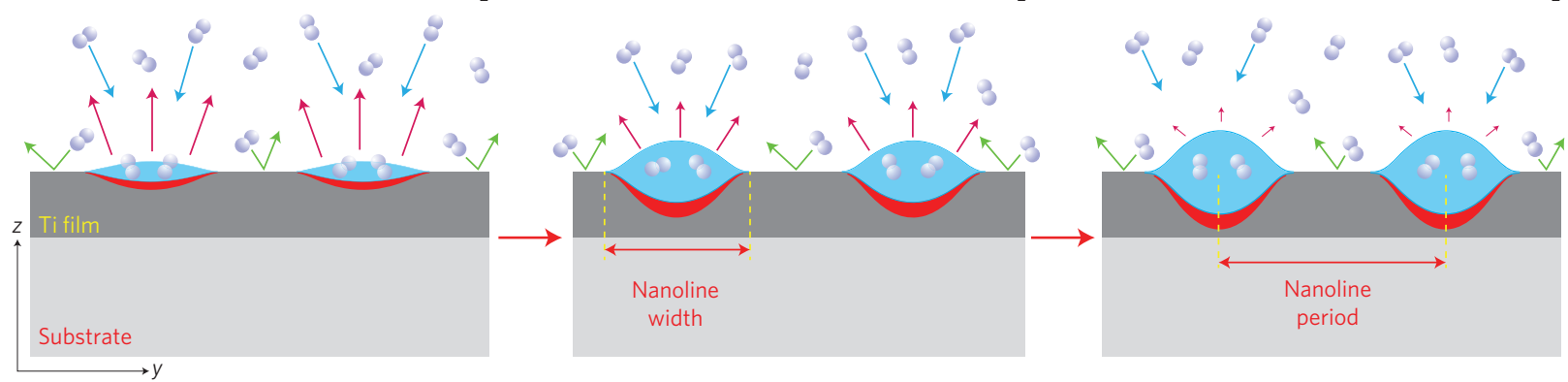

Figure 1 | Conceptual model. a, Schematic of the nanostructure formation process as a laser beam is scanned over the surface. $\mathbf{b}$, Schematic showing a cross-sectional view of the surface, depicting the deceleration of the growth process due to negative feedback.

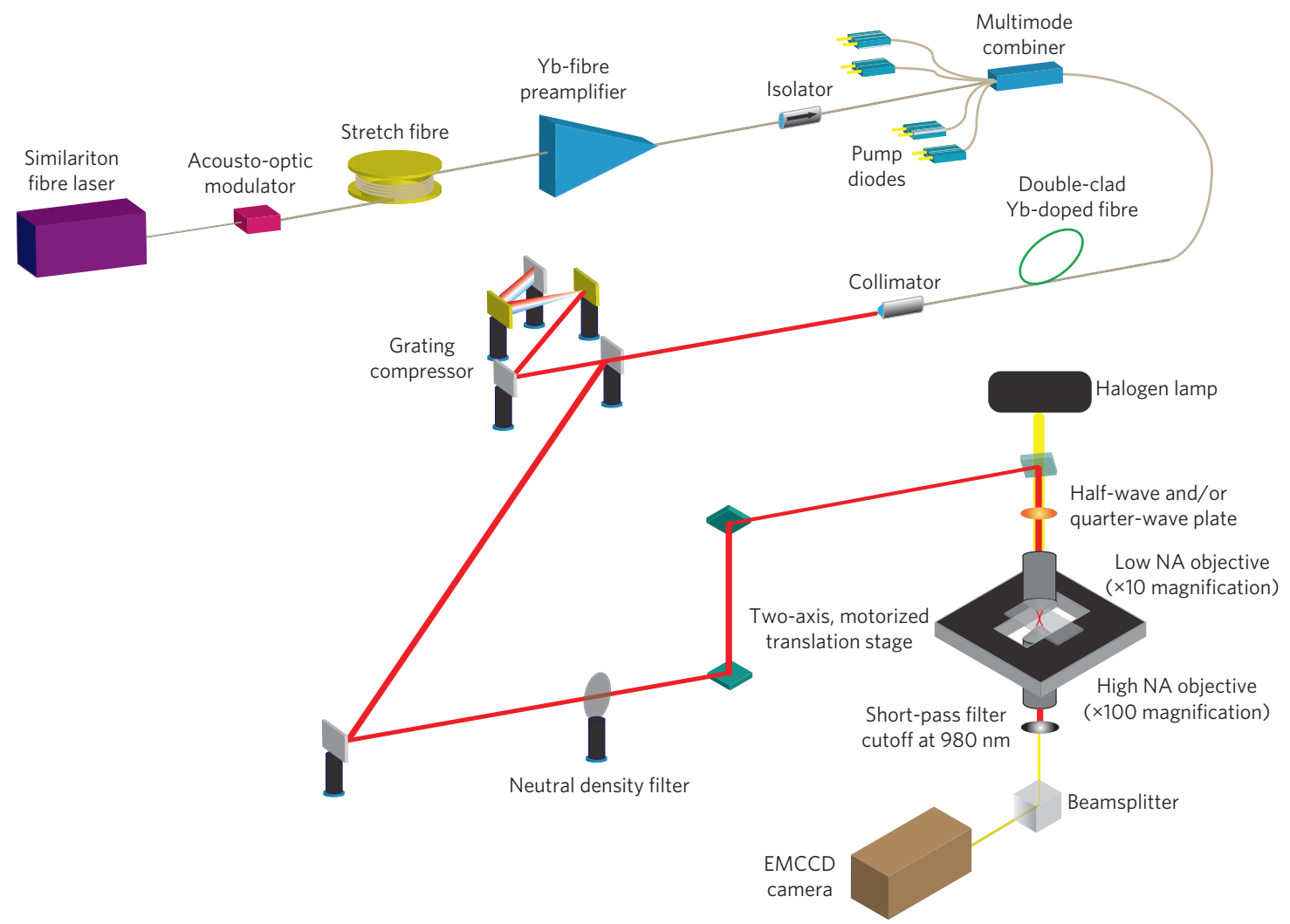

Figure 2 | Experimental set-up. An amplified fibre laser is coupled to a custom-built, computer-controlled optical microscope set-up. EMCCD, electronmultiplying charge-coupled device; NA, numerical aperture. 
a
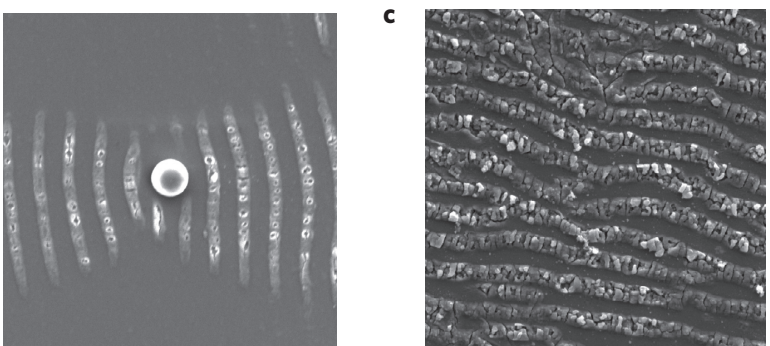

b

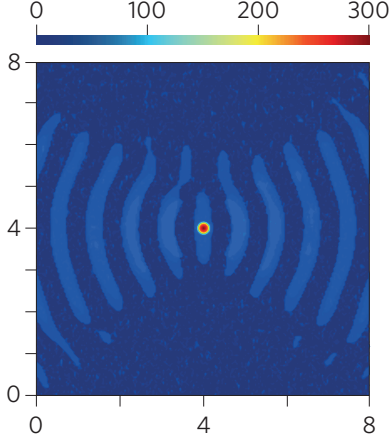

300 d 0

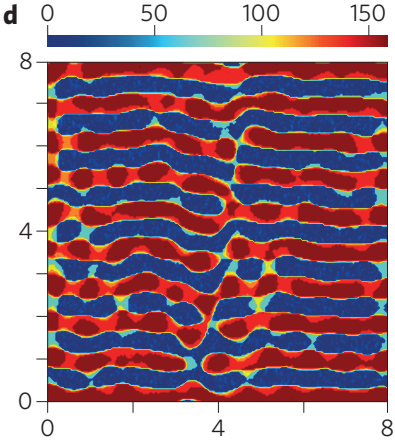

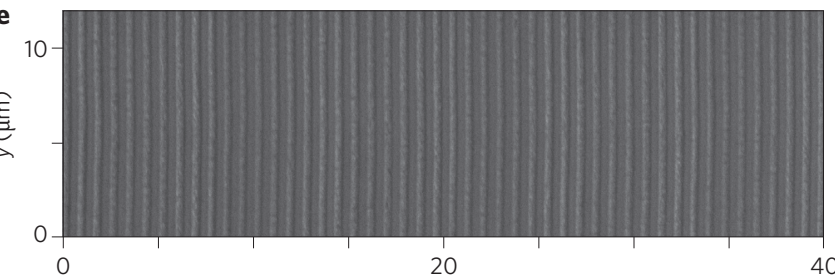

$x(\mu \mathrm{m})$

f

50

100

150

200

10
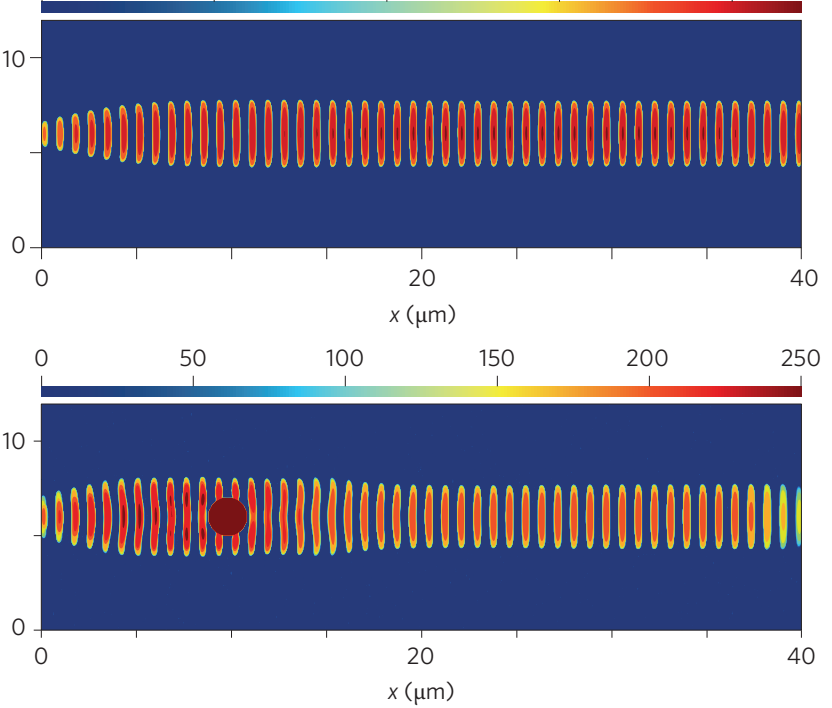

Figure 3 | Nanostructure formation dynamics. a,b, SEM image of the experimental results (a) and numerical simulation results (b) of nanostructures formed around an isolated scatterer by a few, high-energy pulses with linear polarization. c,d, SEM image of the experimental results (c) and numerical simulation results (d) of nanostructures obtained with a large and stationary laser beam. e, SEM image of uniform nanostructures obtained by scanning a small laser beam. f, Numerical simulation results of nanostructures obtained by scanning a small laser beam. $\mathbf{g}$, Numerical simulation results showing robustness of the nanostructure formation against a defect, showing minor distortion and quick subsequent recovery. Colour bars indicate height in nanometres.

forming metal oxide $\left(\mathrm{TiO}_{2}\right)$ of an amount that is proportional to the laser-activated metal (titanium) or available $\mathrm{O}_{2}$ at that point, whichever is smaller. Here, we simply refer to this controlled transformation as ablation, because similar physical processes underlie it, even though the metal is not removed, but is chemically transformed. As a result of the ablation threshold, no processing of the surface should occur between the nanolines, where partially destructive interference leads to the total intensity being below the ablation threshold. The presence of this threshold, which was confirmed experimentally (Fig. 3; Supplementary Section 'Experimental evidence for the threshold for intensity'), is the main source of nonlinearity.

When scanning a small-diameter beam, the nanostructures are created sequentially, with existing structures creating new structures, similar to the toppling of dominoes. This enables the formation of extremely uniform nanostructures (experimental and simulated results are shown in Fig. $3 e$ and $f$, respectively). Moreover, it is possible to tile indefinitely large areas with nanostructures, without a discernible reduction in long-range uniformity when using a small laser beam. We scan the beam along a line, then shift the beam laterally while still preserving a partial overlap with the previous point, and then scan again parallel to the line of the scan (with partial overlap being maintained all along the way with the first line of the scan). This can be visually observed in Supplementary Movie S1, where the red disk represents the beam location and verified experimentally (Supplementary Movie S2). Further evidence of the role of nonlocal feedback lies in the fact that the new structures form a tilted front and the nanolines become distorted into a wavy pattern at the end of each scan line due to incomplete nonlocal feedback.
The nanostructure formation mechanism exhibits a significant degree of robustness against distinct types of perturbations. First, the resultant field at any point is formed collectively by the entire surrounding area, so the contributions of isolated defects or rough patches on the surface are easily overwhelmed. When a defect is placed along the beam path (under conditions otherwise the same as in Fig. 3f), the nanolines suffer only minor distortions (Fig. 3g). Defects encountered in Supplementary Movie S2 provide experimental confirmation. Second, Supplementary Movie S2 shows that the beam focus was not maintained well during scanning due to the poor mechanical stability of our set-up. However, key features, such as nanoline period and width, are independent of laser power (see Supplementary Section 'Insensitivity of the nanostructure features to laser power and exposure time' for direct experimental confirmation). Because of this insensitivity, a partial loss of focus during scanning is inconsequential. In fact, we found the standard and Allan deviations of the nanoline period of this structure to be $0.9 \mathrm{~nm}$ and $0.14 \mathrm{~nm}$, respectively (for details see Supplementary Section 'Characterization of the uniformity of the nanostructures'). Third, as a result of the negative feedback mechanism, the growth of the nanostructures saturates at a given height. Even minutes-long exposure to a stationary beam or multiple scans of the laser over the same area have no discernible effect (Supplementary Movie S4). Robustness against a range of perturbations is a coveted feature of nonlinear systems ${ }^{5}$ that is extremely difficult to achieve in strictly linear systems.

A diverse range of nanostructures have been fabricated using this approach. A photograph of nanostructures covering a $3 \mathrm{~mm}^{2}$ area, fabricated on a thin and flexible glass slide, is presented in Fig. 4a. 

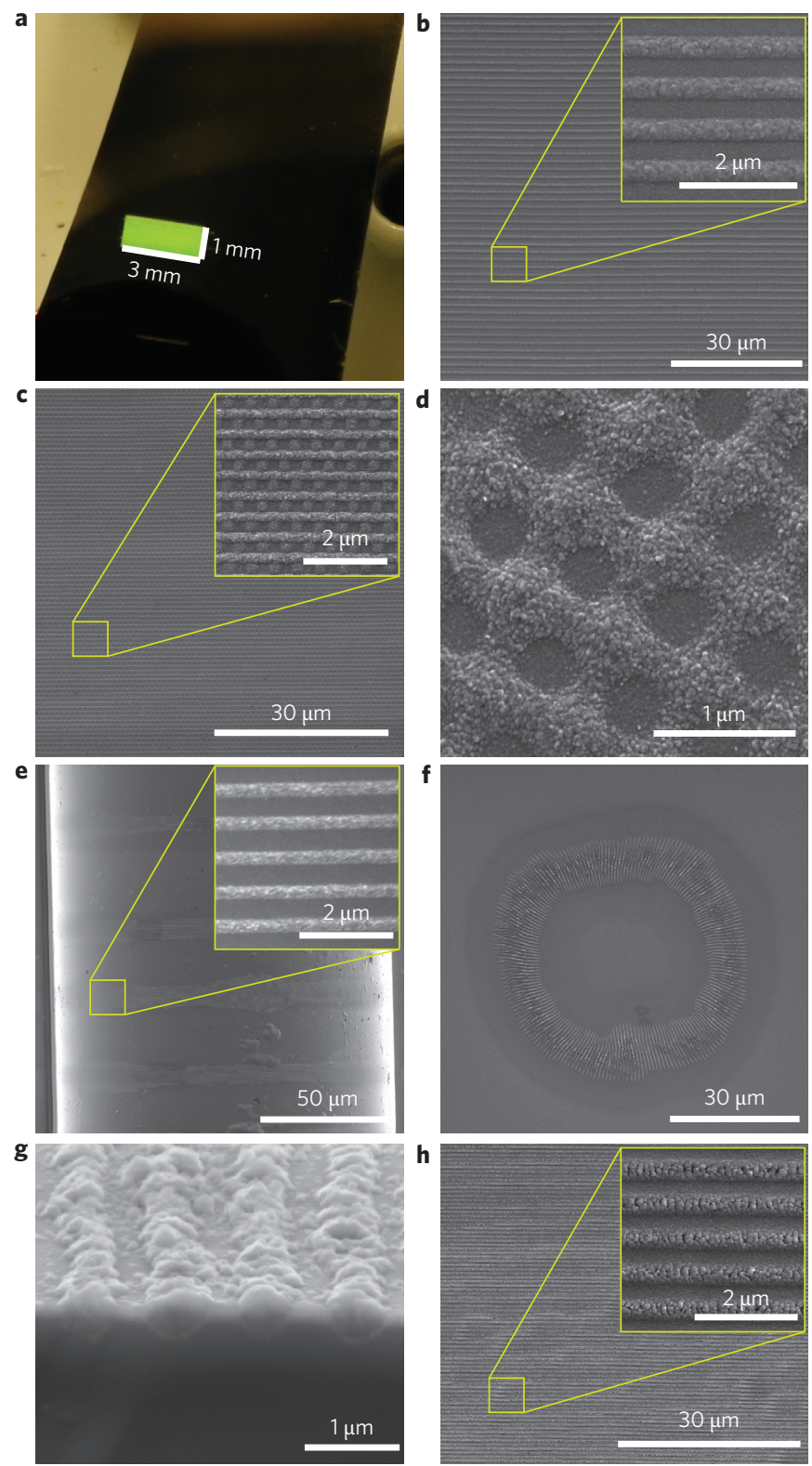

Figure 4 | Examples of fabricated nanostructures. a, Photograph of nanostructures covering an area of $1 \mathrm{~mm} \times 3 \mathrm{~mm}$ (colouration is due to diffraction of room light). $\mathbf{b}$, SEM image of a portion of the same structure. c, SEM image of mesh structure obtained by two scans of the beam with orthogonal polarizations. d, Nanocircles (diameter, $370 \mathrm{~nm}$ ) obtained with circularly polarized light. $\mathbf{e}$, Nanostructures fabricated on the side of a titanium-coated optical fibre, demonstrating the capability to fabricate on non-planar surfaces. $\mathbf{f}$, Circular pattern of nanostructures obtained by rotation of the polarization direction. $\mathbf{g}$, Cross-sectional SEM image of structures created on a thin film of titanium on a silicon substrate, showing imprinting of the nanostructures to the underlying substrate. h, Nanostructures obtained on a tungsten film over a glass substrate.

A scanning electron microscope (SEM) image of a section of the same structure is shown in Fig. 4b. A mesh structure of titanium dots surrounded by a mesh of $\mathrm{TiO}_{2}$ is obtained by scanning with a linearly polarized beam, followed by a second pass with $90^{\circ}$-rotated polarization (Fig. 4c). Using circularly polarized light, a regular array of nanocircles is obtained (Fig. 4d, Supplementary Movie S4). An important advantage of this method is the capability to create these structures on non-planar surfaces due to the insensitivity of the process to variations in laser intensity. This is in clear contrast to conventional lithography techniques. As a demonstration, we scanned the beam over a titanium-coated optical fibre (125 $\mu \mathrm{m}$ diameter), creating structures despite the very strong surface curvature (Fig. 4e). Synchronously rotating the polarization during a circular scan results in optical resonator-like patterns (Fig. 4f), which could be interesting given the high index of $\mathrm{TiO}_{2}$. The structures can be imprinted on a substrate such as silicon, where the titanium film is used only as a transfer material (Fig. 4g). If desired, titanium can be selectively etched away afterwards. Although we focused on titanium in this work, our approach should be applicable to other materials. Indeed, Fig. 4h shows tungsten oxide structures fabricated on a tungsten surface.

To conclude, we report a simple, low-cost and high-speed method based on exploiting naturally occurring feedback mechanisms for the creation of metal-oxide nanostructures with femtosecond pulses. We have demonstrated periodic nanostructures covering areas measuring square millimetres with $\sim 1 \mathrm{~nm}$ longrange uniformity on bulk and thin metal films on flexible substrates, as well as on the surface of an optical fibre, proving that non-planar surfaces can be processed. These special features, primarily the ability to process curved surfaces, are nominally not achievable with conventional techniques. In addition, our technique exhibits a substantial degree of robustness against defects and perturbations. This constitutes another demonstration that unique technological capabilities can emerge naturally by exploitation of nonlinear photonic systems ${ }^{7}$. Although we optimized the process for the oxidation of titanium and tungsten under a regular atmosphere, many metals, semiconductors and dielectrics have been shown to support LIPSS formation ${ }^{15-19}$. In principle, any of these materials can be subjected to a variety of chemical reactions under a suitable atmosphere to fabricate nanostructures from a virtually inexhaustible list of material compositions. The fabricated nanostructures can find applications in plasmonics ${ }^{21}$, plasmonic nanolithography ${ }^{22}$, photon detection ${ }^{23}$, nanophotonics ${ }^{24}$, memristors ${ }^{25}$ and metallic nanostructures $^{26}$ for nanoelectronics, control of cell behaviour through patterned surfaces ${ }^{27}$ and in low-cost fabrication of highly ordered $\mathrm{TiO}_{2}$ structures, which have been shown to significantly increase the efficiency of dye-sensitized solar cells ${ }^{28}$.

\section{Methods}

Laser set-up. The laser source was a home-built $\mathrm{Yb}$-doped fibre laser, operating at a central wavelength of $1,060 \mathrm{~nm}$ and generating pulses with up to $1 \mu \mathrm{J}$ energy at $1 \mathrm{MHz}$, which can be compressed to $100 \mathrm{fs}$ (ref. 20). The powers incident on the samples ranged from $100 \mathrm{~mW}$ to $1 \mathrm{~W}$. The pulse-to-pulse power stability was on the order of $0.05 \%$ (measured from $3 \mathrm{~Hz}$ to $250 \mathrm{kHz}$ ).

Integrated laser microscope set-up. The pulses from the laser were coupled into a modified inverted microscope (Nikon Eclipse Ti) using a dichroic mirror with high reflectivity for $1 \mu \mathrm{m}$ and high transmission of visible light. The focused spot size was set to $\sim 12 \mu \mathrm{m}$ (full-width at half-maximum intensity) in most experiments. Beam positioning was achieved by moving the sample on a dual-axis step-motor stage (Thorlabs, MAX203) with a repeatability of $\sim 1 \mu \mathrm{m}$. The samples were illuminated with a halogen source, and imaging was carried out with a $\times 100$ oil-immersion objective. The images were recorded with an electron-multiplying charge-coupled device camera (Andor, Luca).

Theoretical model. The semi-phenomenological three-step model was based on an integro-difference equation with decoupled timescales. The laser beam was modelled as a Gaussian beam centred at a point $\left(x_{0}, y_{0}\right)$ on the surface. When the $n$th pulse of the pulse train was incident, it scattered from the surface protrusions and depressions, described by the local height of titanium, $h_{n}(x, y)$. The total laser intensity was calculated for every point $(x, y)$ on the surface by integrating the contributions from the surrounding surface elements. Ablation occurs on a picosecond timescale, much faster than thermal diffusion, which would otherwise smear out the nanoscale localization of the absorbed laser energy. At points where the laser intensity exceeded the ablation threshold, titanium was ablated from its top surface down to the point where the intensity dropped below the ablation threshold The amount of $\mathrm{O}_{2}$ available was calculated based on the thickness of the $\mathrm{TiO}_{2}$ layer at that point. Ablated titanium and $\mathrm{O}_{2}$ react readily on a timescale much slower than the ablation process, but faster than the arrival of the next pulse $(1 \mu \mathrm{s})$. The amount of $\mathrm{TiO}_{2}$ formed was determined by the amount of ablated titanium and $\mathrm{O}_{2}$, whichever was smaller. The surface profile was then updated as $h_{n+1}(x, y)$. The entire 
process was repeated for the $(n+1)$ th pulse with an updated beam position $\left(x_{0}, y_{0}\right)$. A full account of the model is given in Supplementary Section 'Detailed description of the theoretical model'.

Received 13 November 2012; accepted 9 September 2013; published online 20 October 2013

\section{References}

1. Bhalla, U. S. \& Iyengar, R. Emergent properties of networks of biological signaling pathways. Science 283, 381-387 (1999).

2. Hopfield, J. J. Neural networks and physical systems with emergent collective computational abilities. Proc. Natl Acad. Sci. USA 79, 2554-2558 (1982).

3. Ferrell, J. E. Jr. Self-perpetuating states in signal transduction: positive feedback, double-negative feedback and bistability. Curr. Opin. Cell Biol. 14, 140-148 (2002).

4. Mandelbrot, B. How long is the coast of Britain? Statistical self-similarity and fractional dimension. Science 156, 636-638 (1967).

5. Kitano, H. Biological robustness. Nature Rev. Genet. 5, 826-837 (2004).

6. Haus, H. A. Theory of mode locking with a fast saturable absorber. J. Appl. Phys. 46, 3049-3058 (1975).

7. Segev, M., Soljačić, M. \& Dudley, J. M. Fractal optics and beyond. Nature Photon. 6, 209-210 (2012).

8. Dudley, J. M., Finot, C., Richardson, D. J. \& Millot, G. Self-similarity in ultrafast nonlinear optics. Nature Phys. 3, 597-603 (2007).

9. Barth, J. V., Costantini, G. \& Kern, K. Engineering atomic and molecular nanostructures at surfaces. Nature 437, 671-679 (2005).

10. Ito, T. \& Okazaki, S. Pushing the limits of lithography. Nature 406, 1027-1031 (2000).

11. Sreekanth, K. V., Chua, J. K. \& Murukeshan, V. M. Interferometric lithography for nanoscale feature patterning: a comparative analysis between laser interference, evanescent wave interference, and surface plasmon interference. Appl. Opt. 49, 6710-6717 (2010).

12. Whitesides, G. M. Self-assembly at all scales. Science 295, 2418-2421 (2002).

13. Gattass, R. R. \& Mazur, E. Femtosecond laser micromachining in transparent materials. Nature Photon. 2, 219-225 (2008).

14. Birnbaum, M. Semiconductor surface damage produced by ruby lasers. J. Appl. Phys. 36, 3688-3689 (1965).

15. Temple, P. \& Soileau, M. Polarization charge model for laser-induced ripple patterns in dielectric materials. IEEE J. Quantum Electron. 17, 2067-2072 (1981).

16. Sipe, J. E., Young, J. F., Preston, J. S. \& van Driel, H. M. Laser-induced periodic surface structure I: theory. Phys. Rev. B 27, 1141-1154 (1983).

17. Bonch-Bruevich, A. M., Libenson, M. N., Makin, V. S. \& Trubaev, V. V. Surface electromagnetic waves in optics. Opt. Eng. 31, 718-730 (1991).

18. Sun, Q., Liang, F., Vallée, R. \& Chin, S. L. Nanograting formation on the surface of silica glass by scanning focused femtosecond laser pulses. Opt. Lett. 33, 2713-2715 (2008).
19. Bonse, J., Krüger, J., Höhm, S. \& Rosenfeld, A. Femtosecond laser-induced periodic surface structures. J. Laser Appl. 24, 042006 (2012).

20. Kalaycioglu, H., Oktem, B., Senel, C., Paltani, P. P. \& Ilday, F. Ö. Microjouleenergy, $1 \mathrm{MHz}$ repetition rate pulses from all-fiber-integrated nonlinear chirpedpulse amplifier. Opt. Lett. 35, 959-961 (2010).

21. Atwater, H. A. \& Polman, A. Plasmonics for improved photovoltaic devices. Nature Mater. 9, 205-213 (2010).

22. Srituravanich, W., Fang, N., Sun, C., Luo, Q. \& Zhang, X. Plasmonic nanolithography. Nano Lett. 4, 1085-1088 (2004).

23. Konstantatos, G. \& Sargent, E. H. Nanostructured materials for photon detection. Nature Nanotech. 5, 391-400 (2010).

24. Juan, M. L., Righini, M. \& Quidant, R. Plasmon nano-optical tweezers. Nature Photon. 5, 349-356 (2011)

25. Strukov, D. B., Snider, G. S., Stewart, D. R. \& Williams, R. S. The missing memristor found. Nature 453, 80-83 (2008).

26. Didiot, C., Pons, S., Kierren, B., Fagot-Revurat, Y. \& Malterre, D. Nanopatterning the electronic properties of gold surfaces with self-organized superlattices of metallic nanostructures. Nature Nanotech. 2, 617-621 (2007).

27. Flemming, R. G., Murphy, C. J., Abrams, G. A., Goodman, S. L. \& Nealey, P. F. Effects of synthetic micro- and nano-structured surfaces on cell behavior. Biomaterials 20, 573-588 (1999).

28. Kang, T.-S., Smith, A. P., Taylor, B. E. \& Durstock, M. F. Fabrication of highlyordered $\mathrm{TiO}_{2}$ nanotube arrays and their use in dye-sensitized solar cells. Nano Lett. 9, 601-606 (2009).

\section{Acknowledgements}

The authors acknowledge support from the Scientific and Technological Research Council of Turkey (TÜBITAK; grant nos 106G089 and 209T058) and a Distinguished Young Scientist award from the Turkish Academy of Sciences (TÜBA). The authors thank G. Ertaş for help with Raman spectroscopy.

\section{Author contributions}

B.Ö. and I.P. conducted the experiments and analysed the data. I.P., S.I. and F.Ö.I. developed the theoretical model and I.P. performed the simulations. I.P., B.Ö., A.R., S.Y. and M.E. constructed the laser microscope set-up. H.K., B.Ö. and A.R. constructed the laser system.

\section{Additional information}

Supplementary information is available in the online version of the paper. Reprints and permissions information is available online at www.nature.com/reprints. Correspondence and requests for materials should be addressed to F.Ö.I.

\section{Competing financial interests}

The authors declare no competing financial interests. 